\title{
Successful implementation of spacer treatment guideline for acute asthma
}

\author{
C V E Powell, G R Maskell, M K Marks, M South, C F Robertson
}

\begin{abstract}
Aims-To develop and implement an evidence based guideline for the treatment of acute asthma using a metered dose inhaler and spacer combination. Methods-Defined strategies were used for the development and implementation of a guideline, assessed by a prospective, descriptive, study using notes review, and patient, nursing, and medical staff telephone contact. The setting was a tertiary referral hospital in Victoria, Australia with 25000 yearly admissions, and asthma accounting for about $7 \%$ of total. The first 200 children and families to use the guideline after its introduction were evaluated. Results-A total of 191 (95.5\%) children were treated according to the guideline. Six $(3.0 \%)$ children were given nebulisers appropriately based on severity; five $(2.5 \%)$ were given nebulisers at parental or child choice; and four (2.0\%) who did not have severe asthma, received nebulised treatment inappropriately.

Conclusions-Successful implementation of a new evidence based guideline can be achieved using specific strategies for promoting the application of research findings in the clinical arena.

(Arch Dis Child 2001;84:142-146)
\end{abstract}

Keywords: acute asthma; spacers; evidence based guideline; change in practice

Department of Emergency Medicine, Royal Children's Hospital, Flemington Road, Parkville, Victoria 3052, Australia

C V E Powell

Department of Thoracic Medicine, Royal Children's

Hospital

C F Robertson

Hospital Support Unit, Royal Children's

Hospital

G R Maskell

Department of Paediatrics, University of Melbourne,

Australia

M K Marks

M South

Correspondence to: Dr Powell

powellc@cryptic.rch. unimelb.edu.au

Accepted 9 August 2000 change the practice of the major paediatric teaching hospital which is perceived as the community leader in child health. In spite of the weight of evidence in favour of the pMDI/ spacer combination, we recognised that a comprehensive approach would be required for the effective promotion of change in practice.

There are many barriers to the successful implementation of research findings into the clinical arena. ${ }^{5}$ Promoting a change in policy or clinical guideline using an evidence based approach may be ineffective unless specific strategies are employed. ${ }^{6}$

Our aims were to develop an evidence based guideline (best practice) for the treatment of acute asthma in children using a pMDI/spacer combination and to successfully implement the change into current medical practice in a major paediatric teaching hospital.

\section{Methods}

SETTING AND PREVIOUS PRACTICE

The Royal Children's Hospital ( $\mathrm{RCH}$ ) provides primary and secondary level care to children in Melbourne and tertiary level care to the children of Victoria and beyond. Annually, there are 60000 attendances to the ED; about 4500 per year are for acute exacerbations of asthma. There are 25000 admissions to hospital per year, with asthma accounting for about $7 \%$ of total. Mean length of stay for asthma in $\mathrm{RCH}$ is 39 hours. $^{7}$

Previous practice in the ED was to assess the severity of asthma and then treat with oxygen driven, nebulised doses of a $\beta_{2}$ agonist, three doses, 20 minutes apart for the first hour. Depending on severity, nebulised ipratropium bromide could be added and steroids given with all treatments based on weight and/or age of the child. If the child responded to this treatment there would be a reduction of the frequency of nebulised treatment, with regular review over the next few hours before a decision was made to admit or not. If the child deteriorated, continuous nebulised $\beta_{2}$ agonists with corticosteroids, intravenous $\beta_{2}$ agonists, and/or aminophylline may be administered. The child may then be admitted to the paediatric intensive care unit (PICU) or the ward. ${ }^{8}$ All patients seen in the ED were given this treatment before the new guideline was introduced. Initially on the ward nebuliser treatment would continue until it was considered that the child had responded well enough to be changed over to the spacer/pMDI combination.

STEPS TAKEN TO ACHIEVE CHANGE

A multidisciplinary group was convened, composed of medical members from the emergency department, general paediatric, respiratory, and intensive care teams, along with the nurs- 
Table 1 Characteristics of the first 200 children seen in the emergency department (ED) using the new guideline (\% in parentheses)

\begin{tabular}{lccc}
\hline & $\begin{array}{c}\text { IEA } \\
(n=130)\end{array}$ & $\begin{array}{c}\text { FE/PA } \\
(n=70)\end{array}$ & $\begin{array}{l}\text { Total } \\
(n=200)\end{array}$ \\
\hline Admitted on this occasion & $46(35.4)$ & $35(50.0)$ & $81(40.5)$ \\
Previous admission (within the past 12 months) & $25(19.2)$ & $41(58.6)$ & $66(33.0)$ \\
Previous ED attendance (within the past 12 months) & $35(26.7)$ & $\begin{array}{c}42(60.0) \\
7(38.5)\end{array}$ & $\begin{array}{c}7(3.0) \\
\text { Previous intensive care unit admission }\end{array}$ \\
\hline
\end{tabular}

IEA, infrequent episodic asthma; FE, frequent episodic asthma; PA, persistent asthma.

ing staff. Initial representation at the planning stage from all teams in the hospital involved with the care of asthma was felt to be crucial if the change in policy was to be successful. ${ }^{9} \mathrm{~A}$ number of team members undertook a detailed review of the current published evidence of best practice and the guideline was developed (see Appendix). The guideline was then presented at three of the hospital's clinical practice review meetings and distributed to the hospital consultants with a request for feedback. After feedback the guideline was revised to encompass the suggestions, aiming for a consensus.

Once the guideline had been developed, a nursing and medical member of the team facilitated regular interactive educational sessions with the nursing and medical staff on the ward and in the ED over the next three months. These sessions provided an opportunity to ensure all staff had a clear understanding of the evidence for and rationale behind the change in practice and also to contribute to the fine tuning of the guideline. Using workshop techniques we clarified that all staff could practically initiate and effectively administer treatment via a spacer. These sessions were supported by written material, reminding people of the change in practice and by using departmental newsletters as a method of dissemination of information. We then involved other members likely to be affected by the change: pharmacy (both hospital and community); the infection control team (responsible for ensuring adequate guidelines for the sterilisation of spacers); patient support assistants (responsible for the cleaning and preparation of spacers); and the local equipment distribution centre (responsible for providing spacer devices).

To inform the families of children with asthma in the community of the proposed change to treatment, we sought the support of the local newspapers, local and national television, and the state body Asthma Victoria. We developed parent information sheets outlining the rationale for the change, how to use a spacer effectively, and how to care for and clean a spacer; these were deisgned to be issued to parents on arrival at the hospital. Over the three months prior to the change, parents and children attending the ED and those attending paediatric outpatients were informed of the imminent change. We decided on no initial involvement with ambulance staff and general practitioners until we had introduced and evaluated the guideline within the hospital.

Finally the change in policy occurred at the beginning of the year, three weeks before the usual three monthly change over of resident medical staff.

OBSERVATIONAL STUDY

Following the introduction of the new treatment guideline, the next phase was to evaluate the change in practice. We prospectively evaluated the first, 200 consecutive subjects attending the ED, between 11 January 1999 and 11 March 1999. Each week the notes of those subjects who had attended the emergency department the previous week were retrieved and evaluated using a standard proforma. The subjects' notes were identified using the departmental computer generated records searching for a discharge diagnosis of asthma. Demographic and descriptive data were collected concerning the child's asthma. Using international, consensus guideline and classification, ${ }^{8}$ we divided children into two groups: those with infrequent episodic asthma and those with more frequent episodic or persistent asthma. The main outcomes were: whether the new guideline was followed (and reasons why not), the treatment given (during the emergency stay and while on the ward), and final disposition (home, ward, or PICU). If data were not available from the notes, the attending doctor or nurse was contacted and information collected. If data were required from a patient or patient's family, they were either seen on the ward if admitted, or a telephone call was made if the child had been discharged.

\section{Results}

DESCRIPTIVE DATA

Of the 200 children, 123 (65\%) were male; 111 $(55.5 \%)$ were aged less than 5 years old, 61 $(30.5 \%)$ between 5 and 10 years, $23(11.5 \%)$ between 10 and 15 years, and the remaining five $(2.5 \%)$ were older than 15 years. Table 1 illustrates the characteristics of these subjects.

\section{OUTCOMES}

Nebulisers were administered to $15 / 200$ $(7.5 \%)$ children. Of these 15 subjects, nebulisers were used appropriately according to protocol in $6 / 200(3.0 \%)$; four had been admitted to PICU and treated with continuous nebulised treatment; and two had deteriorated on spacer treatment and then recovered sufficiently to avoid admission to PICU, once commenced on continuous nebulised treatment. Table 2 shows the various explanations for treatment with nebulised medication in the 15 subjects.

\section{Discussion}

This new guideline led to a change in the way we treat asthma in this hospital. Although there are still many questions unanswered about the use of spacers in acute asthma, we felt the strength of the published evidence should drive the change in practice. There were clearly a number of issues, such as salbutamol dosage or use of ipratropium bromide with spacers, where we have had to make level $\mathrm{V}$ evidence recommendations. Thus, although we use the 
Table 2 Explanation for non-adherence to the spacer guideline

\begin{tabular}{|c|c|c|c|}
\hline & $\begin{array}{l}I E A \\
(n=130)\end{array}$ & $\begin{array}{l}F E / P A \\
(n=70)\end{array}$ & $\begin{array}{l}\text { Total } \\
(n=200)\end{array}$ \\
\hline \multicolumn{4}{|c|}{ Appropriate use of nebulised treatment } \\
\hline Severity/PICU admission & 3 & 1 & 4 \\
\hline $\begin{array}{l}\text { Deterioration on spacer } \\
\text { treatment }\end{array}$ & 0 & 2 & 2 \\
\hline \multicolumn{4}{|l|}{ Patient choice } \\
\hline Parental request & 1 & 2 & 3 \\
\hline Child choice & 0 & 1 & 1 \\
\hline Child distress & 1 & 0 & 1 \\
\hline \multicolumn{4}{|c|}{ Non-adherence to guidelines } \\
\hline Medical/nursing decision & 4 & 0 & 4 \\
\hline
\end{tabular}

IEA, infrequent episodic asthma; FE, frequent episodic asthma; PA, persistent asthma.

term "evidenced based" guidelines - as with most guidelines, they could be termed "best practice" guidelines.

We have judged the change in management of asthma in our hospital as successful and acceptable; six $(3.0 \%)$ children were given nebulisers appropriately, according to the guideline based on severity. Five $(2.5 \%)$ were given nebulisers at parental or child choice and only four $(2.0 \%)$ received nebulised treatment in error or inappropriately. We conclude that the guideline was followed appropriately in $95.5 \%$ of children. Only a small minority of parents found it unacceptable and asked for nebulised treatment and only a small minority of staff felt it necessary to change back to nebulisers for unclear reasons, but not for reasons of severity.

One could argue that this was an audit study; before introducing the new guideline all patients initially received their treatment via a nebuliser; following our intervention, practice changed so that the data we presented are our post audit findings. We are in no doubt that practice has changed and that it would not have happened without our intervention. Although published evidence for spacer treatment has been available for over a decade, nebulised treatment remained the mainstay of treatment for acute asthma within our establishment and continues to be in other institutions.

Implementing research findings into practice is not an easy process. Despite published evidence supporting the use of spacers and pMDI for acute asthma, the "nebuliser culture" had to be overcome both in hospital medical and nursing staff and also in parents and families of children with asthma. ${ }^{4}$

There are well defined steps that should be taken to promote the clinical uptake of research findings. ${ }^{9}$ Once an appreciable gap has been identified between research findings and practice, a clear appropriate guideline should be developed. Ensuring a critical appraisal of the evidence using current published metaanalyses $^{1}$ and individual papers ${ }^{2}{ }^{3}$ is imperative in order to produce a valid, acceptable, and generalisable guideline. ${ }^{9}$ Involving key players is the next part of the development of the message. Initially there should be a core multidisciplinary team of medical and nursing staff followed by development and refinement of the guideline with the key people who will be using and implementing the guideline. In our care, after a number of presentations at medical meetings, electronic mail contacts, and using the feedback form, the various specialists most involved agreed on the guideline. This was crucial; a study from Leicester in the UK showed failure to introduce a similar protocol and one of the main reasons cited was lack of initial agreement on the guideline from all the specialties involved. ${ }^{10}$ Thus all those people within our organisation, who would have to implement the change were involved in developing the strategies right from the beginning. ${ }^{9}$

There are many potential barriers to change within an organisation. ${ }^{59}$ Nursing and medical staff may rely on obsolete knowledge, be biased towards a particular treatment, hold individual beliefs and attitudes based on previous adverse experience of innovation, and lack motivation. All these may prevent successful implementation of a new evidence based guideline. Patients and parents will also be subject to similar beliefs as well as cultural or perceptual influences affecting their demands for care. Limitations of time, financial resources, and inappropriate educational activities may also be a problem. Identifying these and attempting to address these issues were the next stage of the process. It is clear that activities such as didactic lectures, distribution of educational materials, clinical practice guidelines, or electronic publications are unlikely to promote behavioural changes among health professionals. ${ }^{6}$ Consistently effective interventions are interactive educational meetings such as workshops, multifaceted processes made up of regular meetings, feedback, reminders using local consensus, and developing a guideline with the people who are going to implement and use the guideline. ${ }^{6}$

We ran interactive workshops, focusing on the nurses in the ED and on ward and the junior medical staff; these were the staff who were going to use the guideline and we felt it important that they should have some "ownership" of the protocol. We led meetings with staff to explain the rationale, the guideline, and the problems we expected. Allowing staff members to voice their objections and make suggestions allowed us to shape the guideline further. One of our difficulties was to make sure we discussed the guideline with all nursing staff, particularly those on duty at night. Two of the four patients who were treated with nebulisers inappropriately were given them at night. We foresaw potential problems with parental and child expectation. ${ }^{11}$ Our strategy was to try to prepare families before introducing the change as described above including appearances by medical staff on national and local television highlighting the planned changes. Only four families objected to the use of a spacer in this study, so we consider our efforts in involving and preparing parents and children as successful. We did not attempt to assess cost effectiveness. However, other studies have shown that using spacers is cheaper than using nebulis$\mathrm{ers}^{4}{ }^{12}$ and we are currently assessing this aspect of our change in practice. We did not formally assess the length of time it takes to administer 
medication through a spacer; some studies have suggested that the use of spacers may reduce the child's length of stay in the ED compared to nebulisers. ${ }^{13}$

Once the guideline had been implemented the final stage of promoting research findings was to audit the change in practice, and share the data with those involved. These data have now been used to feedback to parents, children, and medical and nursing staff. We consider that the key to the successful introduction of this guideline was considerable planning and following a proven, structured strategy for implementation of research evidence into clinical practice.

We thank all the staff and patients involved with this implementation.

\section{Appendix}

Treatment for acute paediatric asthma in the department of emergency medicine in the first hour using spacer holding devices

(1) CRITICAL ASTHMA

(defined as episode severe enough to require intensive care)

Use oxygen, continuous nebulised salbutamol along with intravenous steroids, salbutamol, and then aminophylline.

\section{(2) SEVERE ASTHMA}

(notable evidence of recession and increase work of breathing with possible oxygen desaturation and agitation)

Use oxygen if necessary, oral or intravenous steroids, and inhaled medication administered via spacer device and pressurised metered dose inhaler (pMDI) every 20 minutes for the first hour:

Age less than 6 years

$600 \mu \mathrm{g}$ (6 puffs) salbutamol

$80 \mu \mathrm{g}$ (2 puffs) ipratropium

Age greater than 6 years

$1.2 \mathrm{mg}$ (12 puffs) salbutamol

$160 \mu \mathrm{g}$ (4 puffs) ipratropium

(3) MODERATE ASTHMA

(mild/moderate evidence of recession and increased work of breathing, normal oxygen saturation, and fully alert)

Oral steroids and inhaled medication administered via spacer device and pMDI every 20 minutes for the first hour:

Age less than 6 years

$600 \mu \mathrm{g}$ (6 puffs) salbutamol

Age greater than 6 years

$1.2 \mathrm{mg}$ (12 puffs) salbutamol

(4) MILD ASTHMA

(no recession or evidence of increased work of breathing, normal oxygen saturation, and fully alert)

Possible steroids and inhaled medication administered via spacer device and pressurised metered dose inhaler; may only be required once:

Age less than 6 years

$600 \mu \mathrm{g}$ (6 puffs) salbutamol

Age greater than 6 years

$1.2 \mathrm{mg}$ (12 puffs) salbutamol

(pMDI used with one actuation at a time with four or five normal tidal breaths in between each actuation).

1 Cates CJ. Comparison of holding chambers and nebulisers for beta-agonists in acute asthma. A systematic review of ranfor beta-agonists in acute asthma. A systematic review of ran-
domised control trials. (Cochrane library). Update software. domised c
1999.
2 Kerem E, Levison H, Schuh S, et al. Efficacy of albuterol dministered by nebuliser versus spacer device in children. administered by nebuliser vers $1993 ; 123: 313-17$.

3 Chou KJ, Cunningham SJ, Crain EF. Metered dose inhalers with spacers vs nebulisers for paediatric asthma. Arch Paediatr Adolesc Med 1995;149:201-5.

4 Dewar AL, Stewart A, Cogswell JJ, ConnettGJ. A randomised controlled trial to assess the relative benefits of large volume spacers and nebulisers to treat acute asthma in hospital. Arch Dis Child 1999;80:421-3.

5 Haynes B, Haines A. Barriers and bridges to evidence based clinical practice. BMF 1998;317:273-6.

6 Bero LA, Grilli R, Grimshaw JM, Harvey E, Oxman AD, Thomson MA. Closing the gap between research and practice: an overview of systematic reviews and interventions to promote the implementation of research findings. BMF 1998;317:465-8.

7 South M. Reduction in length of stay for acute asthma associated with the introduction of case-mix funding. Med $\mathcal{F}$

8 Warner JO, Naspitz CK. Third International Pediatric Consensus statement on the management of childhood asthma. International Pediatric Asthma Consensus Group. Pediatr Pulmonol 1998;25:1-17.

9 Haines A, Donald A. Making better use of research findings. $B M \mathcal{F}$ 1998;317:72-5.

10 Dunbar H, Barry PW. The use of metered dose inhalers and spacer devices in the treatment of acute severe childhood asthma-changing clinical practice. Eur Respir f 1998;12 (suppl):339.

11 Connett GJ, Warde C, Wooler E, Lenney W. Audit strategies to reduce hospital admissions for acute asthma. Arch Dis Child 1993;69:202-5.

12 Leversha AM, Campanella SG, Aickin RP, Asher MI. Costs and effectiveness of spacer versus nebulizer in young children with moderate and severe asthma. F Pediatr 2000; 136:497-502.

13 Chou K, Cunningham S, Crain E. Metered-doses inhalers with spacers vs. nebulisers for pediatric asthma. Arch Pediatr Adolesc Med 1995;149:201-5.

\section{Commentary}

In the second century $\mathrm{AD}$ Ptolemy the Greek astronomer and mathematician postulated that the sun rotated around the earth. His theory remained unquestioned for 1200 years. In the year of his death, Copernicus (1473-1543) published his Copernican System showing clearly that Ptolemy was wrong and that in fact the earth and other planets orbited the sun. Galileo (1564-1642) agreed with Copernicus but was forced by the Inquisition to recant his support of the Copernican System almost a century after it had been scientifically proven.

It was the best part of a century from when HG Wells predicted man would land on the moon until the event finally took place. Thank goodness that today we accept changes more readily than we did in the past-or do we?

For the past 20 years grant giving bodies supporting medical studies have not only required assurance on the quality of the science and the appropriateness of the ethics but request researchers to explain how the new scientific results will be disseminated. Researchers nonchalantly suggest they will present their findings at national and international meetings and publish papers in peer reviewed medical journals. But is this enough? What is the rate of uptake of new practices following publication in peer reviewed journals?

Even today, studies undertaken in Europe are believed with reluctance in the USA (and vice versa) and there remains an egotistical feeling that the study was not carried out as well as "we" would have done it and it doesn't really apply to "our" patients. Scepticism is fine and the history of medical research is littered with studies which have later been disproved. However, when a number of studies from numerous countries all point in the same direction it strikes me as folly if these do not institute a universal change in practice. 
The benefits of spacer devices over nebulisers in acute childhood asthma may not be as earth shattering as whether the sun or the earth is the centre of the solar system but it addresses an important issue in one of the commonest childhood disorders of our time.

How many of us can honestly say that spacer devices have mainly taken the place of nebulisers in our emergency rooms and in our paediatric wards?

For this reason I think the paper by Powell and his colleagues is immensely important. It shows, in an exemplary way, what managerial steps need to be taken to adopt scientifically proven research results. For true consensus to occur much explanation and discussion is needed. Didactic decisions will inevitably result in failure because colleagues and their views have not been treated with respect.

The paper by Bero and colleagues ${ }^{1}$ is also well worth reading for anyone wishing to undertake similar changes. We may be told that we are living in a world of ever increasing and rapid change, but in reality few of these changes we readily accept at face value and if we are not careful, conflict ensues.

In the paediatric respiratory world perhaps I could ask all readers of $A D C$ : (1) Do you use once daily intravenous gentamicin or still believe three times daily is better? (2) Do you try to restrict trainee doctors obtaining chest $x$ rays in acute asthma? (3) Do you still use beta agonists, inhaled corticosteroids, or ipratropium bromide in acute viral bronchiolitis? (4) Do you have strict criteria for the use of antibiotics in chest infections, the majority of which are viral in origin? (The decision to institute antibiotic therapy can often be delayed until a subsequent consultant ward round, provided that clear protocols are in place) (5) Do you allow patients under the age of 8 years to use breath actuated devices when it has been shown they are unlikely to inhale significant amounts into their lungs?

I suspect we all have a long way to go in optimising delivery of care as shown by well constructed and well executed research studies. We can do far worse than to follow the recommendations of Powell and colleagues.

\section{WARREN LENNEY}

Academic Department of Child Health City General

Hospital Stoke-on-Trent, UK

1 Bero LA, Grilli R, Grimshaw JM, et al. Closing the gap between research and practice: an overview of systemic reviews and interventions to promote the implementation of research findings. BMF 1998;317:465-8.

\section{End of the line for cromoglycate?}

Inhaled disodium cromoglycate has been used as maintenance treatment for asthma for over 30 years and is recommended in current guidelines. Nevertheless, in clinical practice over the last 10 years it has been largely replaced by inhaled steroids. Now a systematic review by workers in the Netherlands (MJA Tasche and colleagues. Thorax 2000;55:913-20) may be the coup de grâce.

The review included 24 randomised controlled trials of disodium cromoglycate in children. Overall, methodology was assessed as weak, especially as regards treatment compliance, selection and inclusion, and statistics and analysis. Funnel plots indicated publication bias with missing small, negative studies. Treatment effects on wheeze and cough were small and tolerance intervals for both included zero. The more recent studies showed results less favourable to disodium cromoglycate. The authors conclude that disodium cromoglycate has not been proved to be better than placebo in the maintenance treatment of childhood asthma and that its promotion as a first line prophylactic treatment is no longer justified.

ARCHIVIST 